\title{
The Complex Dynamics of Wishful Thinking: The Critical Positivity Ratio
}

\author{
Nicholas J. L. Brown \\ Strasbourg, France \\ Alan D. Sokal \\ New York University and University College London \\ Harris L. Friedman \\ Saybrook University and University of Florida
}

July 15, 2013

Published in American Psychologist 68, xxx-xxx (2013)

http://dx.doi.org/10.1037/a0032850

(c) 2013 American Psychological Association

This article may not exactly replicate the final version published in the APA journal. It is not the copy of record.

Running Head: The critical positivity ratio

\section{Author note}

Nicholas J. L. Brown, Strasbourg, France; Alan D. Sokal, Department of Physics, New York University and Department of Mathematics, University College London; Harris L. Friedman, Graduate College of Psychology and Humanistic Studies, Saybrook University and Department of Psychology, University of Florida.

We wish to thank Kate Hefferon and Nash Popovic for their encouragement, and Jean Bricmont, Andrew Gelman, Steven Pinker, and Colin Sparrow for helpful comments on an early draft of this manuscript. The authors, of course, bear full and sole responsibility for the content of this article.

Correspondence concerning this article should be addressed to Alan D. Sokal, Department of Physics, New York University, 4 Washington Place, New York, NY 10003. E-mail: sokal@nyu.edu 


\begin{abstract}
We examine critically the claims made by Fredrickson and Losada (2005) concerning the construct known as the "positivity ratio." We find no theoretical or empirical justification for the use of differential equations drawn from fluid dynamics, a subfield of physics, to describe changes in human emotions over time; furthermore, we demonstrate that the purported application of these equations contains numerous fundamental conceptual and mathematical errors. The lack of relevance of these equations and their incorrect application lead us to conclude that Fredrickson and Losada's claim to have demonstrated the existence of a critical minimum positivity ratio of 2.9013 is entirely unfounded. More generally, we urge future researchers to exercise caution in the use of advanced mathematical tools such as nonlinear dynamics and in particular to verify that the elementary conditions for their valid application have been met.
\end{abstract}

Keywords: Positivity ratio, broaden-and-build theory, positive psychology, nonlinear dynamics, Lorenz system. 
The "broaden-and-build" theory (Fredrickson, 1998, 2001, 2004) postulates that positive emotions help to develop broad repertoires of thought and action, which in turn build resilience to buffer against future emotional setbacks. Fredrickson and Losada (2005) took the broaden-and-build theory a step further, by proposing that an individual's degree of flourishing could be predicted by that person's ratio of positive to negative emotions over time, which they termed the "positivity ratio" (p. 678).

On its own, the positivity ratio as propounded by Fredrickson and Losada (2005) is not a particularly controversial construct; indeed, there is a long history of looking at ratios (e.g., Bales, 1950) and non-ratio indices (e.g., Bradburn, 1969) relating positive to negative emotions. However, Fredrickson and Losada took matters considerably farther, claiming to have established that their use of a mathematical model drawn from nonlinear dynamics provided theoretical support for the existence of a pair of critical positivity-ratio values (2.9013 and 11.6346) such that individuals whose ratios fall between these values will "flourish," while people whose ratios lie outside this ideal range will "languish." The same article purported to verify this assertion empirically, by demonstrating that among a group of college students, those who were "languishing" had an average positivity ratio of 2.3 , while those who were "flourishing" had an average positivity ratio of 3.2 .

The work of Fredrickson and Losada (2005) has had an extensive influence on the field of positive psychology. This article has been frequently cited, with the Web of Knowledge listing 322 scholarly citations as of April 25, 2013. Fredrickson and Kurtz (2011, pp. 41-42), in a recent review, highlighted this work as providing an "evidence-based guideline" for the claim that a specific value of the positivity ratio acts as a "tipping point beyond which the full impact of positive emotions becomes unleashed" (they now round off 2.9013 to 3). An entire chapter of Fredrickson's popular book (2009, Chapter 7) is devoted to expounding this "huge discovery" (p. 122), which has also been enthusiastically brought to a wider audience by Seligman (e.g., 2011a, pp. 66-68; 2011b). In fact, the paperback edition of Fredrickson's book (2009) is subtitled "Top-Notch Research Reveals the 3-to-1 Ratio That Will Change Your Life." 
It is worth stressing that Fredrickson and Losada (2005) did not qualify their assertions about the critical positivity ratios in any way. The values 2.9013 and 11.6346 were presented as being independent of age, gender, ethnicity, educational level, socioeconomic status or any of the many other factors that one might imagine as potentially leading to variability. Indeed, Fredrickson and Losada went so far as to assert (pp. 678, 684, 685) that the same critical minimum positivity ratio of 2.9013 applies to individuals, couples, and groups of arbitrary size (see also Fredrickson, 2009, pp. 133-134). And yet, the idea that any aspect of human behavior or experience should be universally and reproducibly constant to five significant digits would, if proven, constitute a unique moment in the history of the social sciences. It thus seems opportune to examine carefully the chain of evidence and reasoning that led to these remarkable conclusions.

Fredrickson and Losada (2005) based their assertions concerning the critical positivity ratios on previous articles by Losada (1999) and Losada and Heaphy (2004) in which the Lorenz equations from fluid dynamics were "applied" to describe the changes in human emotions over time. The major part of the present paper is devoted, therefore, to a critical examination of the three subject articles, in chronological order. We shall demonstrate that each one of the three articles is completely vitiated by fundamental conceptual and mathematical errors, and above all by the total absence of any justification for the applicability of the Lorenz equations to modeling the time evolution of human emotions. (Furthermore, although the second and third articles rely entirely for their validity on the presumed correctness of their predecessors — which, as we shall demonstrate, is totally lacking — we nevertheless invite the reader, at each stage, to assume for the sake of argument the correctness of the preceding $\operatorname{article(s);~in~this~way~we~are~able~to~explain~more~clearly~the~independent~flaws~of~each~of~the~}$ three subject articles.) We conclude that Fredrickson and Losada's (2005) claims concerning the alleged critical values of the positivity ratio are entirely unfounded.

\section{A brief introduction to differential equations and nonlinear dynamics}

Although the three subject articles rest on a purported application of differential equations - a branch of mathematics in which most psychologists are unlikely to be expert - 
to social-psychological data, none of the three articles provides its readers with any more than a vague explanation of the mathematics on which those articles rely. It therefore seems appropriate to begin this critical analysis by giving a brief (and we hope pedagogical) introduction to differential equations, explaining what they are and when they can be validly used. While we appreciate that it is unusual to find this type of material in a psychology journal, we nevertheless encourage even the less mathematically-inclined reader to attempt to follow our explanations as far as possible, and to consult a mathematician or physicist friend for help if needed (and to verify that our arguments are correct).

\section{What are differential equations?}

Differential equations are employed in the natural and social sciences to model phenomena in which one or more dependent variables $x_{1}, x_{2}, \ldots, x_{n}$ evolve deterministically as a function of time $(t)$ in such a way that the rate of change of each variable at each moment of time is a known function of the values of the variables at that same moment of time 1

Let us unpack this definition slowly, beginning with the case in which there is a single dependent variable $x$.

One dependent variable. We consider a situation in which both the independent variable $t$ ("time") and the dependent variable $x$ can be treated as continuous quantities; we furthermore assume that $x$ varies smoothly as a function of $t$. First-year calculus then defines the instantaneous rate of change of $x$, conventionally written $d x / d t$. A (first-order)

\footnotetext{
${ }^{1}$ More precisely, in this paper we shall be concerned exclusively with what mathematicians call ordinary differential equations: these are the simplest type of differential equation, and are also the type that is employed in the three subject articles. For completeness let us mention that there also exist other types of differential equations, including partial differential equations (in which there are two or more independent variables, rather than the single independent variable $t$ considered here) and stochastic differential equations (in which the time evolution involves explicit randomness). Some of the statements made here concerning the properties of ordinary differential equations require modification in connection with these other types of equations. For instance, the requirement of deterministic evolution (items DE3 and VA3 below) manifestly does not apply to stochastic differential equations.
} 
differential equation for the function $x(t)$ is an equation of the form

$$
\frac{d x}{d t}=F(x)
$$

where $F$ is a known (i.e., explicitly specified) function. What this says, in words, is that the rate of change of $x$ at any moment of time is precisely the function $F$ applied to the value of $x$ at that same time. More specifically, an equation of this form is saying that:

(DE1) Both time $(t)$ and the dependent variable $(x)$ can be treated as continuous quantities.

(DE2) $x$ changes smoothly over time (i.e., it does not jump).

(DE3) $x$ evolves deterministically (i.e., there is no randomness).

(DE4) The rate of change of $x$ at any given moment of time depends only on the value of $x$ itself (i.e., not on some additional variables), and only on the value of $x$ at that same moment of time (i.e., not on the values in the past).

(DE5) The rate of change of $x$ at time $t$ is exactly $F(x(t))$, where $F$ is an explicitly specified function.

The mathematical model of the phenomenon under study thus consists of Equation 1 together with the specification of the function $F$.

Example 1. Consider a bank account with continuously compounded interest. Then the amount of money in the account $(x)$ increases with time $(t)$ according to the differential equation

$$
\frac{d x}{d t}=r x
$$

where $r$ is the interest rate. This has the form of Equation 1 with $F(x)=r x$. This same equation describes the cooling of a coffee cup, the decay of radioactive atoms, and a vast number of other physical, biological, and social phenomena. (In some of these applications, $r$ is a negative number.) Equation 2 is an example of a linear differential equation, because the function $F(x)=r x$ is linear (i.e., doubling $x$ causes $F(x)$ to precisely double).

Equation 2 happens to have a simple solution, namely $x(t)=x_{0} e^{r t}$, where $x_{0}$ is the account balance at time 0 , and $e \approx 2.71828$ is the base of natural logarithms. This formula 
illustrates an important general principle: the solution of a differential equation is completely determined by the initial conditions, that is, the value(s) of the dependent variable(s) at time 0 .

It should be stressed that most differential equations do not have simple solutions that can be explicitly written down; rather, the solutions have to be studied numerically, by computer. Nevertheless, the solution at arbitrary time $t$ is always determined, at least in principle, from the initial conditions, even though it may not be given by any simple explicit formula.

Example 2. Consider a population $x$ of some species living in a limited territory, with a maximum sustainable population $X_{\max }$. Then a plausible (though of course extremely oversimplified) model for the growth of this population is given by the differential equation

$$
\frac{d x}{d t}=r x\left(1-\frac{x}{X_{\max }}\right)
$$

where $r$ is some positive number, which has the form of Equation 1 with $F(x)=r x(1-$ $\left.x / X_{\max }\right)$. Equation 3 is an example of a nonlinear differential equation, because here the function $F$ is nonlinear (i.e., doubling $x$ does not cause $F(x)$ to precisely double).

Of course, population is not, strictly speaking, a continuous variable, since there is no such thing as a fractional person. This would appear to conflict with principle DE1 above. But if the population $x$ is large (e.g., millions of people), then only a negligible error is made by treating the population as if it were a continuous variable.

Several dependent variables. Let us now consider the case in which we have several dependent variables. We assume once again that both the independent variable $t$ and the dependent variables $x_{1}, \ldots, x_{n}$ can be treated as continuous quantities, and that $x_{1}, \ldots, x_{n}$ vary smoothly as a function of $t$. Then a system of (first-order) differential equations for the functions $x_{1}(t), \ldots, x_{n}(t)$ is a system of equations of the form

$$
\begin{aligned}
\frac{d x_{1}}{d t} & =F_{1}\left(x_{1}, \ldots, x_{n}\right) \\
& \vdots \\
\frac{d x_{n}}{d t} & =F_{n}\left(x_{1}, \ldots, x_{n}\right)
\end{aligned}
$$


where $F_{1}, \ldots, F_{n}$ are specified functions. The model is constituted by the system of Equations 4 together with the specification of the functions $F_{1}, \ldots, F_{n}$.

Example 3. Lorenz (1963), building on work by Saltzman (1962), introduced the following system of differential equations as a simplified model of convective flow in fluids:

$$
\begin{aligned}
& \frac{d X}{d \tau}=-\sigma X+\sigma Y \\
& \frac{d Y}{d \tau}=r X-Y-X Z \\
& \frac{d Z}{d \tau}=-b Z+X Y
\end{aligned}
$$

Here the independent variable $\tau$ is a dimensionless variable that is proportional to time $t$. The dependent variables $X, Y$, and $Z$ are also dimensionless, and represent various aspects of the fluid's motion and its temperature gradients. (In the solutions studied by Lorenz, $X$ and $Y$ oscillate between positive and negative values, while $Z$ stays positive.) Finally, $\sigma, b$, and $r$ are positive dimensionless parameters that characterize certain properties of the fluid and its flow; in particular, $r$ measures (roughly speaking) the strength of the tendency to develop convection.

For future reference, let us explain what is meant by "dimensionless." A quantity is called dimensionful if its numerical value depends on an arbitrary choice of units. For instance, lengths (measured in meters or furlongs or ...) are dimensionful, as are times and masses. By contrast, a quantity is called dimensionless if its numerical value does not depend on a choice of units. For instance, the ratio of two lengths is dimensionless, because the units cancel out when forming the ratio; likewise for the ratio of two times or of two masses. Physical laws are best expressed in terms of dimensionless quantities, since these are independent of the choice of units; this explains why Lorenz (1963) rescaled his equations as he did. In particular, Lorenz's variable $\tau$ equals $t / T$, where $T$ is a particular "characteristic time" in the fluid-flow problem. 


\section{Nonlinear dynamics and chaos}

The mathematical field of nonlinear dynamics — popularly known as chaos theory - is founded on the observation that simple equations can under some circumstances have extremely complicated solutions. In particular, fairly simple systems of nonlinear differential equations can exhibit sensitive dependence to initial conditions: that is, small changes in the initial conditions can lead to deviations in the subsequent trajectory that grow exponentially over time. Such systems, while deterministic in principle, can be unpredictable in practice beyond a limited window of time; the behavior can appear random even though it is not. We refer the reader to Lorenz (1993), Stewart (1997) and Williams (1997) for sober nontechnical introductions to nonlinear dynamics, and to Strogatz (1994) and Hilborn (2000) for introductions presuming some background in undergraduate mathematics and physics.

The Lorenz system, revisited. The Lorenz system (Equations 5 above) illustrates nicely some of the concepts of nonlinear dynamics. Firstly, this system has a fixed point at $X=Y=Z=0$ : if the system is started there, it stays there forever. (Physically, this fixed point corresponds to a fluid at rest.) For $r<1$ it turns out that this fixed point is stable: if the system is started near the fixed point, it will move towards the fixed point as time goes on. For $r>1$ this fixed point is unstable: if started near the fixed point, the system will move away from it.

For $r>1$ there is another pair of fixed points, at $X=Y= \pm \sqrt{b(r-1)}, Z=r-1$. (Physically, these fixed points correspond to a steady-state convective flow.) It turns out that these fixed points are stable for $r<r_{\text {crit }}$ and unstable for $r>r_{\text {crit }}$, where $r_{\text {crit }}=$ $\sigma(\sigma+b+3) /(\sigma-b-1)$ and we assume $\sigma>b+1$.

What happens for $r>r_{\text {crit }}$ ? Lorenz (1963) investigated the trajectories numerically and found that they tend to a butterfly-shaped set now known as the Lorenz attractor. This set is a fractal: it is neither two-dimensional (a surface) nor three-dimensional (a volume) but something in-between. Moreover, the trajectories near the attractor exhibit sensitive dependence to initial conditions (i.e., they are chaotic) 2

2 Actually, the Lorenz attractor is "born" at a value $r_{\mathrm{A}}$ slightly less than $r_{\text {crit }}$; for $r_{\mathrm{A}}<r<r_{\text {crit }}$, both 
One final remark: Mathematicians studying the Lorenz system typically (though not always) fix $\sigma$ and $b$ and vary $r$. Saltzman (1962) chose the illustrative values $\sigma=10$ and $b=8 / 3$; then Lorenz (1963) followed him, as have most (though not all) workers ever since. But there is nothing magical about these values; indeed, any other values within a fairly wide range would produce qualitatively similar behavior (Sparrow, 1982, pp. 179-184).

\section{When can differential equations validly be applied?}

When used properly, differential equations constitute a powerful tool for modeling timedependent phenomena in the natural and social sciences. But a number of preconditions must be met if such an application is to be valid. In order to apply differential equations to a specific natural or social system, one must first:

(VA1) Identify and define precisely the variables that specify the state of the system at a given moment of time. Per principles DE1 and DE2 (above), these variables must be continuous (or at least approximately so), not discrete, and they must evolve smoothly in time (i.e., without jumps). These variables need not be directly observable quantities; they can also be (hypothetical or hidden) latent variables, which are postulated to affect the observable quantities in specified ways.

(VA2) Give reasons why these variables can be assumed to evolve by themselves, without significant effect from other variables not taken into account in the model (cf. principle DE4).

(VA3) Give reasons why these variables can be assumed to evolve deterministically, at least to a good approximation (cf. principle DE3).

(VA4) Give reasons why these variables can be assumed to evolve according to a differential equation (at least to a good approximation): that is, that the rate of change at each moment of time depends only on the values of the variables at that same moment of time (cf. principle DE4).

(VA5) Find the specific differential equation giving (at least approximately) that evolution: that is, find the functions $F_{1}, \ldots, F_{n}$ and give arguments justifying them for the specific 
system under study. In particular, if the functions $F_{1}, \ldots, F_{n}$ involve unspecified constants (such as the constants $r$ and $X_{\max }$ in Examples 1 and 2, or $\sigma, b$, and $r$ in Example 3) and the behavior of the system depends in a significant way on those constants, then arguments must be given to justify the particular values that are given to those constants.

Only after the conditions VA1-VA5 have been fulfilled does it make sense to apply the theory of differential equations in general, or the theory of nonlinear dynamics in particular, to the natural or social phenomenon under study.

It goes without saying that these conditions are not easy to fulfill; and they become more difficult to fulfill, the more complex is the system under study. Consequently it is not surprising that most of the valid applications of nonlinear dynamics have arisen in physics and chemistry, where one can sometimes find systems that are sufficiently simple and isolated so that one can (a) identify a small number of relevant variables that evolve by themselves, and (b) write down the equation describing (at least to a reasonable degree of approximation) their evolution. See also Ruelle (1994), Kellert (1995), and Sokal and Bricmont (1998, Chapter 7) for some pertinent cautions concerning the applicability of nonlinear dynamics to the modeling of real-world phenomena.

There is also a valid "shortcut" approach to modeling using differential equations: carry out VA1 strictly, but skip VA2-VA5 and simply guess (by whatever means) the functions $F_{1}, \ldots, F_{n}$; then obtain empirical data that are sufficiently powerful to constitute strong evidence that VA2-VA5 hold for the system under study (though one may not yet understand why).

To summarize: In all cases it is necessary to carry out VA1 strictly and to give evidence that VA2-VA5 hold in one's application; but there are two alternatives concerning the type of evidence one supplies. In the "standard" approach one gives theoretical arguments that VA2-VA5 hold, while in the "shortcut" approach one gives empirical evidence that VA2-VA5 hold. Of course, if one is able to provide compelling evidence of both types, so much the better. 


\section{Analysis of Losada (1999)}

We begin this section by summarizing Losada's (1999) experimental setup and briefly pointing out some serious deficiencies therein. These deficiencies would suffice, by themselves, to render Losada's empirical work of limited or no scientific value; but they are of only minor importance compared to the principal abuse in Losada's article, which is the purported application of the Lorenz equations to describe the changes in human emotions over time. In the preceding section we listed five criteria, VA1-VA5, all of which must be met if an application of differential equations is to be valid. Here we shall demonstrate that none of these criteria have been met by Losada's work, except arguably a small part of VA1. We shall also demonstrate that the conditions for validity of the "shortcut" approach have not been met either.

\section{Losada's experiments}

Losada (1999) described a series of experiments that he conducted during the 1990s while he was the director of a laboratory ("Capture Lab") run by the U.S. computer services corporation EDS. Within this laboratory, 60 business teams were studied during meetings, while observers positioned behind one-way mirrors analyzed and coded their verbal communications; some details of the procedures used were described by Losada and Markovitch (1990). These coded data form the entire empirical basis of all the subsequent work allegedly leading to the theoretical derivation of the critical minimum positivity ratio using a nonlinear-dynamics model.

Losada's (1999) article followed few of the conventions that would normally be expected from a piece of scholarship published in a scientific journal. It presented very little primary data, and the experimental design, construction of models, and interpretation of results were made with little or no justification. Indeed, some aspects of the methods and results of the Capture Lab experiments were described for the first time only in subsequent articles or books, while many other crucial aspects remain obscure to this day.

Many important details of the "business teams," and of their meetings that were observed in the laboratory setting, are not clear. Losada (1999, p. 188) stated that, because of 
capacity constraints in the Capture Lab, each of the 60 observed teams was composed of exactly eight members; but he did not say whether any otherwise-eligible teams had a "realworld" membership larger (or smaller) than eight, and if so, whether they were truncated (or expanded) for the experiment or simply excluded from participation. Losada also did not indicate how long the meetings lasted, though Fredrickson (2009, p. 123) finally reported, informally, that these were "hour-long" meetings. Not even the most basic demographic information, such as the sex ratio or the mean ages of the participants, was provided. Above all, neither Losada (1999) nor either of the two later articles discussed any possible effects on the participants of being observed and recorded during their workplace meetings. We consider these to be substantial omissions for an article describing an empirical experiment in psychology, especially one whose results have been used with such portentous theoretical consequences.

The "speech acts" of the members of the business teams during their meetings were coded according to three bipolar dimensions: "positivity-negativity," "other-self," and "inquiryadvocacy" (Losada, 1999, p. 181). It is not clear whether each speech act was coded according to all three dimensions, or to only one.

Having rated teams as "high performance," "medium performance," or "low performance" based on business indicators, Losada (1999, p. 180) next proceeded to analyze the teams' "degree of connectivity" or "nexi index," which he defined as "the number of crosscorrelations significant at the .001 level or better that were obtained through the time series analysis of the data generated by coding speech acts at the Capture Lab." This is a crucial piece of information because Losada went on to use its value directly as the control parameter - which he called $c$, and which Lorenz (1963) called $r$ - in the Lorenz equations. Losada gave no precise definition, however, of what he meant by "number of cross-correlations," nor did he specify what statistical test he was using to define the significance level, nor why he chose the particular level of 0.001; note that any other choice would have given a different value for the Lorenz control parameter c. Losada (1999, p. 180) did say vaguely that these cross-correlations "represent sustained couplings or matching patterns of inter- 
locked behaviors among participants throughout the whole meeting." From remarks made later in the article (p. 188) as well as slightly more detailed explanations given in Losada and Heaphy (2004, pp. 747-748, 763) and Fredrickson (2009, p. 124), we tentatively infer that "the number of cross-correlations" might mean the number of ordered pairs $i, j$ of team members $(i \neq j)$ such that the cross-correlation function $C_{i j}(t)$ of their time series meets certain unspecified criteria 3 The maximum possible number of such "nexi" for an eight-person group would therefore be $8 \times 7=564$ The rounded average nexi for high-performance, medium-performance and low-performance teams were 32, 22, and 18, respectively (Losada, 1999, p. 180).

\section{Losada's use of differential equations}

Having examined some of the deficiencies in Losada's (1999) description of the empirical part of his research, we now move on to the fundamental problem in the article: namely, the use of differential equations to model the time evolution of human emotions in general, or interactions within business teams in particular. We organize our analysis according to the criteria VA1-VA5 (set forth previously) for the valid application of differential equations. This analysis divides naturally into two parts: the general use of differential equations (VA1VA4), and the specific use of the Lorenz system (VA5).

VA1. Losada (1999, p. 182) stated that the variables $X, Y, Z$ represent, respectively,

\footnotetext{
${ }^{3}$ Losada and Heaphy (2004, p. 763, note 2) cited Losada, Sánchez, and Noble's (1990) concept of a "group interaction diagram" - a sort of directed graph in which the nodes are the group members and the arrows indicate cross-correlations that are nonzero and statistically significant under some unspecified statistical tests - as providing "a graphical representation of nexi". This supports our tentative inference about the probable intended meaning of Losada's (1999) term "number of cross-correlations".

4 The alternative guess that "nexi" refer to unordered pairs of team members is contradicted by the fact that the maximum possible number of such "nexi" for an eight-person group would be 28, while Losada's high-performance teams had an average nexi index of 32. Note, however, that if "nexi" refer to ordered pairs of team members, then the criteria defining a nexus - whatever they may be, as Losada (1999) has not told us - would have to be time-asymmetric, since $C_{i j}(t)=C_{j i}(-t)$; otherwise we would, de facto, be using unordered pairs after all.
} 
"inquiry-advocacy," "other-self," and "emotional space." Earlier (p. 181) he defined "emotional space" as "the ratio of positivity to negativity." But he did not indicate anywhere in the article whether $X$ and $Y$ are defined as ratios of "inquiry" to "advocacy" and "other" to "self," respectively, or as differences. (In fact, either option leads to severe difficulties invalidating his use of the Lorenz equations, as discussed below.)

Even more importantly, Losada (1999) gave no explanation of how the "speech acts," which are discrete events occurring at discrete moments of time, are to be converted into smoothly varying functions $X(t), Y(t), Z(t)$ of a continuous time variable $t$. Arguably this could be done, at least approximately, by subdividing the one-hour session into time intervals $\Delta t$ that are both (a) long enough so that each interval contains a large number of "speech acts" (so that the discrete ratios or differences could be approximated in a sensible way by a continuous variable $X, Y$, or $Z$ ), and (b) short enough so that the change in $X, Y, Z$ from one interval to the next is small (so that the discrete time variable indexing the intervals could be approximated in a sensible way by a continuous variable $t$, with $X, Y, Z$ varying reasonably smoothly as a function of $t$ ). It is at least conceivable that these two conditions could be simultaneously fulfilled, though we think it unlikely:5

The key problem, however, is not simply that Losada (1999) failed to explain any of this to his reader. The problem is, rather, that there is not the slightest evidence that Losada did any of this. Nowhere in Losada (1999) — or, for that matter, in the two subsequent articles - is there any description of empirical data that have the form of smoothly varying functions $X(t), Y(t), Z(t)$ of a continuous time variable $t$. Indeed, none of the three articles

\footnotetext{
${ }^{5}$ Five years later, Losada and Heaphy (2004, p. 745) finally revealed that "data generated by the coders were later aggregated in one-minute intervals." They indicated that "time series analyses, including the auto-correlation and cross-correlation function, were performed on these aggregated data"; but they did not explain whether (or how) these aggregated data were also used to construct the smoothly varying functions $X(t), Y(t), Z(t)$ whose time evolution is allegedly modeled by the Lorenz system, nor did they present any information whatsoever concerning these time series other than their means (p. 747, Table 1). Curiously, Table 1 presents the means for the ratios in all three categories, although Losada and Heaphy (2004, p. 754) later implied that $X$ and $Y$ might be defined via differences rather than ratios (see the discussion below).
} 
evinces any awareness that a phenomenon describable by a differential equation must involve quantities that vary smoothly as a function of time.

We conclude that Losada (1999) has failed to satisfy the most basic and elementary requirement VA1 for the applicability of differential equations. This alone would invalidate his work.

VA2. Losada (1999) did not give any arguments to support the idea that his variables $X, Y, Z$ evolve by themselves; the question was not even considered. A priori it seems implausible that these three variables should evolve autonomously, without significant influence from other variables that might affect the emotional state of a business meeting.

VA3. Losada (1999) did not give any arguments to support the idea that his variables $X, Y, Z$ evolve deterministically; once again, the question was not even considered. A priori this hypothesis seems even more implausible than the preceding one.

VA4. Losada (1999) did not give any arguments to support the idea that his variables $X, Y, Z$ evolve according to a differential equation, that is, that the rate of change at each moment of time depends only on the values of the variables at that same moment of time (and not on past values). This too is a priori implausible as it is tantamount to assuming that the participants in the meeting have no memory. It is, of course, conceivable that change in emotions is dominated (at least under some circumstances) by the current state of emotions, so that the effect of memory is small; if this were the case and VA1-VA3 were also to hold, then VA4 might be justified as a reasonable approximation. But Losada did not even raise this possibility, much less give any theoretical or empirical evidence to support it.

VA5. Finally, let us suppose, for the sake of argument and contrary to what we have just shown, that Losada (1999) had adequately fulfilled criteria VA1-VA4, that is, that he had precisely defined all his variables and had demonstrated that their time evolution should be governed, at least to a good approximation, by a deterministic first-order differential equation. The question would still remain: What reasons did Losada (1999) give for supposing that the governing equation should be the Lorenz system?

To see the type of reasons that are offered, let us quote in full Losada's (1999) "derivation" 
of his first equation, which corresponds to Equation $5 \mathrm{c}$ above:

Thinking about the model that would generate time series that would match the general characteristics of the actual time series observed at the Capture Lab [this issue will be discussed below], it was clear that it had to include nonlinear terms representing the dynamical interaction among the observed behaviors. One such interaction is that between inquiry-advocacy and other-self. If I call the first $X$ and the second $Y$, their interaction should be represented by the product $X Y$, which is a nonlinear term. I also knew from my observations at the lab, that this interaction should be a factor in the rate of change driving emotional space (which I will call $Z$ ). In addition, I would need a scaling parameter for $Z$. Consequently, the rate of change of $Z$ should be written as

$$
\frac{d Z}{d t}=X Y-a Z,
$$

where $a$ is a scaling parameter that would be held constant. (Losada, 1999, p. 182)

Losada (1999) gave no explanation of why the "interaction" between $X$ and $Y$, and only these, should be significant in determining the rate of change of $Z$ ("positivity/negativity"). It would seem equally plausible that the rate of change of $Z$ might be affected by the "interaction" of $X$ and $Z$, or of $Y$ and $Z$, or of all three variables together. Nor is it explained why this "interaction" should consist of multiplying their values together; indeed, if $X$ and $Y$ are dimensionless quantities (as they must be for the Lorenz system to be valid), any mathematical operation could be used to combine them, such as the square root of the sum of their squares, or the difference of their exponentials, or the product of their cube roots. Finally, it is not explained why the rate of change of $Z$ should contain a term linear in $Z$ but not one in $X$ or $Y$.

Similar considerations apply to Losada's (1999, p. 182) subsequent "derivation" of every term in his three equations. The principal attribute that all of the choices made by Losada have in common is that they correspond to terms in Lorenz's system of differential equations. The reader is left with the feeling of having watched a video clip of a Rubik's Cube being miraculously solved in five seconds, only for it to be revealed at the end that what was 
filmed was an ordered cube being scrambled, with the whole process then being played back in reverse 6

A final problem is posed by Losada's (1999) lack of clarity as to whether his variables $X$ and $Y$ are defined as ratios of "inquiry/advocacy" and "other/self," respectively, or as differences. Either option leads to fundamental contradictions with the Lorenz system. If $X$ and $Y$ are ratios of speech acts (as the analogy with $Z$ would suggest), then they are dimensionless and obviously nonnegative. If $X$ and $Y$ are differences of speech acts - as Losada and Heaphy (2004, p. 754) seemed to imply when discussing the values plotted on the $\mathrm{x}$-axis of their Figure 5 - then they are dimensionful (they would be rates of speech acts per unit time, so that their numerical values would depend on the choice of the unit of time) and take both positive and negative values (depending, e.g., on whether inquiry predominates over advocacy or vice versa). In the Lorenz system, by contrast, $X$ and $Y$ are dimensionless and take both positive and negative values (as Losada's Figures 1-6 clearly show). Therefore, no matter how Losada's (1999) unclarity about the definition of $X$ and $Y$ were to be resolved, those variables would not have the properties assumed in the Lorenz system.

\section{The alleged match between Losada's empirical data and his mathematical model}

Losada (1999, p. 183) asserted that "The time series generated by this model matched all the general characteristics of the time series observed at the lab for each team performance level." However, he did not provide any empirical data showing the time series $X(t), Y(t), Z(t)$ allegedly produced by his lab data; indeed, as discussed earlier, he failed to explain, even in the vaguest terms, how such smoothly varying functions were suppos-

\footnotetext{
${ }^{6}$ Luoma, Hämäläinen, and Saarinen (2008) made considerable effort to connect Losada's (1999) work with their own concept of Systems Intelligence; even so, they felt compelled to observe (p. 760) that "there is no further discussion [of] why these [Lorenz] equations should describe the dynamics of the coded observations of verbal communication. Only very limited explanations are given about the modelling process and the meaning and interpretation of its parameters (see Losada, 1999, p. 182). Thus, the reasoning behind the model equations remains unclear to the reader."
} 
edly constructed from his raw data on "speech acts." Nor did he explicate the nebulous phrase "general characteristics." Losada's (1999) claim that his model matches his data must therefore be taken entirely on faith.

For what it is worth, it seems a priori unlikely that the emotions of business teams composed of normal individuals would oscillate wildly and continuously throughout a onehour meeting in the manner of the Lorenz attractor. For instance, Losada's (1999) Figures 1 and 2 allegedly show the ratio of positivity to negativity (what Losada called "emotional space") for high-performance teams fluctuating repeatedly between a minimum of about 10 and a maximum of about 50 . Both numbers are thoroughly implausible, as is the factor-of-5 swing between them.

It follows that Losada (1999) has also failed to meet the minimal conditions for validity of the "shortcut" approach to modeling using differential equations, for at least four reasons (any one of which would be fatal): the variables are not clearly defined (VA1); the purported empirical verification is weak or nonexistent; it is a priori implausible that the data could match the model even qualitatively, much less quantitatively (VA5); and it is a priori implausible (as noted earlier) that even VA2-VA4 could hold for the system under study.

What Losada (1999) did in Section 5 (pp. 183-188) of his article was simply to run some computer simulations of the Lorenz equations at parameters $\sigma=10, b=8 / 3$, and $r=$ $32,22,18$. To the results of these purely mathematical simulations, shown in his Figures 19, Losada then appended the words "inquiry-advocacy," "other-self," "emotional space," and "connectivity" (or "number of nexi") to the mathematical quantities $X, Y, Z$, and $r$, respectively. He referred to the simulations at $r=32,22,18$ as describing the trajectories of "high performance teams" (p. 183), "medium performance teams" (pp. 187-188), and "low performance teams" (pp. 184-185), respectively; but he presented no data to support this purported identification.

Let us also note that both $r=22$ and $r=18$ lie in the region $r<r_{\mathrm{A}} \approx 24.06$ (see Footnote 2) where the solution of the Lorenz system settles down, as $t \rightarrow \infty$, to a fixed point at $X=Y= \pm \sqrt{b(r-1)}, Z=r-1$. The results for "medium performance teams" and 
"low performance teams" shown in Losada's (1999) Figures 4-9 are thus transient behavior, not indicative of the system's long-term state, and reflective only of Losada's arbitrary (and unspecified) choices of initial conditions and total run length. In particular, Losada's assertion (p. 188) that the $r=22$ data end up in a limit cycle - repeated in Losada and Heaphy (2004, pp. 751, 755, 762), Fredrickson and Losada (2005, pp. 682, 683, 685) and Fredrickson (2009, pp. 126, 129) — is incorrect 7

But let us once again put aside all the foregoing criticisms and suppose, just for the sake of argument and contrary to all evidence, that Losada (1999) had adequately demonstrated that the time evolution of emotions within his teams' business meetings was governed by the Lorenz system. There still arises the question of how the dimensionless parameters $\sigma$, $b$, and $r$ in the Lorenz equations are to be chosen. For $\sigma$ and $b$, Losada (p. 183) simply followed the arbitrary choice made for purely illustrative purposes by Saltzman (1962) and Lorenz (1963), and set $\sigma=10$ and $b=8 / 3$. For the key parameter $r$, Losada (p. 182) set this equal to the number of nexi $(c)$, but without providing any justification for doing so. In fact, choosing $r$ equal to (rather than merely dependent on) the number of nexi is puzzling, since $r$ is a universal control parameter in the Lorenz system, while the number of nexi obviously depends on the size of the business team. Consequently, even if the "results" of the three subject articles were somehow to be scientifically valid (and of course they are not), they would apply at best to the dynamics of eight-person teams, rather than being universal truths about human emotions.

\section{Conclusion}

One can only marvel at the astonishing coincidence that human emotions should turn out

\footnotetext{
7 Although dynamical-systems theory focuses principally on the system's long-time behavior, it should be stressed that the transient behavior is not necessarily without interest. Our goal in the present paragraph is merely to observe that a central thesis of the three subject articles — namely, the parallel between the authors' concepts of low-, medium- and high-performance teams and the mathematical concepts (which indeed pertain to long-time behavior) of fixed points, limit cycles, and strange attractors — is fatally flawed by (among many other things) an erroneous understanding of the behavior of the Lorenz system.
} 
to be governed by exactly the same set of equations that were derived in a celebrated article several decades ago as a deliberately simplified model of convection in fluids, and whose solutions happen to have visually appealing properties. An alternative explanation - and, frankly, the one that appears most plausible to us - is that the entire process of "derivation" of the Lorenz equations has been contrived to demonstrate an imagined fit between some rather limited empirical data and the scientifically impressive world of nonlinear dynamics.

But Losada (1999) goes farther:

An interesting observation that highlights the usefulness of fluid dynamics concepts to describe human interaction arises from the fact that Lorenz chose the Rayleigh number as a critical control parameter in his model. This number represents the ratio of buoyancy to viscosity in fluids. A salient characteristic of my observations of teams at the Capture Lab was that high performance teams operated in a buoyant atmosphere created by the expansive emotional space in which they interacted and that allowed them to easily connect with one another. Low performance teams could be characterized as being stuck in a viscous atmosphere highly resistant to flow, created by the restrictive emotional space in which they operated and which made very difficult for them to connect with one another; hence, their nexi were much lower than the nexi for high performance teams. (Losada, 1999, p. 183)

Let us pass quickly over the notion that Lorenz simply "chose" to use the Rayleigh number in his fluid-dynamics equations (just as Einstein perhaps "chose" to use the speed of light in his equation $E=m c^{2}$ ?) as well as the minor technical error in the second sentence of this paragraph (the ratio of buoyancy to viscosity in fluids is the Grashof number, not the Rayleigh number). Instead, we invite the reader to contemplate the implications of the third and fourth sentences. They appear to assert that the predictive use of differential equations abstracted from a domain of the natural sciences to describe human interactions can be justified on the basis of the linguistic similarity between elements of the technical vocabulary of that scientific domain and the adjectives used metaphorically by a particular observer to describe those human interactions. If true, this would have remarkable implications for 
the social sciences. One could describe a team's interactions as "sparky" and confidently predict that their emotions would be subject to the same laws that govern the dielectric breakdown of air under the influence of an electric field. Alternatively, the interactions of a team of researchers whose journal articles are characterized by "smoke and mirrors" could be modeled using the physics of airborne particulate combustion residues, combined in some way with classical optics.

\section{Analysis of Losada and Heaphy (2004)}

Losada and Heaphy (2004) claimed to extend Losada's (1999) "findings" by demonstrating that the latter's construct of "connectivity" is, in fact, directly arithmetically related to the positivity-negativity $(\mathrm{P} / \mathrm{N})$ ratio. In this section we shall try, as best we can, to follow Losada and Heaphy's (2004) reasoning. For the purposes of this exercise, we invite the reader to suppose, for the sake of argument and contrary to everything that we have just demonstrated, that all of Losada's (1999) "results" are entirely correct; in this way we can highlight (within the limits of the space available to us) the independent deficiencies of logic in Losada and Heaphy's article 8

\section{The redefinition of "emotional space"}

Recall that Losada (1999, p. 181) defined "emotional space" as "the ratio of positivity to negativity." But, as noted above, this definition is blatantly incompatible with Losada's (1999, p. 182) identification of "emotional space" with the Lorenz variable $Z$, since the latter fluctuates (when $\sigma=10, b=8 / 3, r=32$ ) between approximately 10 and 50. Perhaps conscious of this, Losada and Heaphy (2004) opted to change (entirely arbitrarily) the definition of "emotional space":

We know that emotional space is generated by the $\mathrm{P} / \mathrm{N}$ ratio. The scale of the $\mathrm{y}$-axis [note that Lorenz (1963) and Losada (1999) called this $Z$ ] does not represent directly

\footnotetext{
${ }^{8}$ Let us warn the reader that, confusingly, Losada and Heaphy (2004) have interchanged the names of the variables $Y$ and $Z$ compared to the convention employed by Lorenz (1963) and Losada (1999). For clarity we will continue to use Lorenz's (1963) conventions as in Equations 5 above; this must be borne in mind when comparing our formulae with those of Losada and Heaphy (2004).
} 
the $\mathrm{P} / \mathrm{N}$ ratio, but the outcome of the initial value (16) entered into the equation to eliminate the transient (this is a standard procedure in nonlinear dynamics and in modeling in general) and the multiplication by the constant $8 / 3$ (a constant used in all Lorenz system models). By introducing the initial value and multiplying by a constant we are creating an initial emotional space that will stay there increased or decreased by the $\mathrm{P} / \mathrm{N}$ ratio. (Losada \& Heaphy, 2004, p. 754)

This paragraph is somewhat confusing, but let us do our best to decipher it.

In the first sentence, Losada and Heaphy (2004) have replaced Losada's (1999, p. 181) "equal to" with a much vaguer "generated by"; and in the first half of the second sentence they have explicitly denied Losada's definition (but without admitting this forthrightly). What comes next is, however, not merely incorrect but in fact the exact opposite of the truth. The initial conditions (such as Losada's apparent choice $Z_{0}=16$ ) are essentially irrelevant to determining the long-time behavior of the system 9 Depending on what initial condition is chosen, one may have to wait a longer or shorter time (the "transient") before reaching the true long-time behavior (e.g., the Lorenz attractor); but that ultimate behavior (including what Losada and Heaphy call "the scale of the y-axis") is determined solely by the parameters $\sigma, b$, and $r$ in the Lorenz system (Equations 5 above), not by the initial condition. Finally, the remainder of this paragraph — that is, the comment regarding "multiplication by the constant $8 / 3$ " as well as the entire final sentence - obeys no logic that we are able to discern. But it does lead to the apparently desired result: namely, "emotional space" is no longer equal to the $\mathrm{P} / \mathrm{N}$ ratio — despite this being Losada's (1999, p. 181) own definition of the term "emotional space" — but is apparently related to it by some as-yet-unspecified formula involving addition (or subtraction) and multiplication.

Having redefined "emotional space" as something other than the $\mathrm{P} / \mathrm{N}$ ratio, Losada and Heaphy's (2004) next task was to "address the question of whether emotional space is linked

\footnotetext{
${ }^{9}$ At least when there is a unique stable attractor, as is the case for $r=32$. For $r=22,18$ there are two stable fixed points, at $X=Y= \pm \sqrt{b(r-1)}, Z=r-1$, and the initial conditions determine which one of them is reached as $t \rightarrow \infty$.
} 
to connectivity and how emotional space is specifically related to the positivity to negativity ratio" (p. 755). They did this in several steps, as described in the following subsections.

\section{Linking "emotional space" and "connectivity"}

Losada and Heaphy's (2004) first step was to draw attention to the "blank space approximately in the middle of the attractor in each of its wings" (p. 755), which they called the "focus" of the attractor. These "foci" are nothing other than the unstable (for $r>r_{\text {crit }}$ ) fixed

points of the Lorenz equations, which as we saw earlier are located at $X=Y= \pm \sqrt{b(r-1)}$, $Z=r-1$. Losada and Heaphy did not mention this fact, but they did observe empirically that the foci are located at $Z=r-1$ for their three simulations, which they rewrote as $E=c-1$, "where $E$ is emotional space, and $c$ is connectivity (represented by the number of nexi)" (pp. 755-756).

\section{Linking "emotional space" and the positivity-negativity ratio}

Losada and Heaphy's (2004) next step was to link "emotional space" $(E)$ to the $\mathrm{P} / \mathrm{N}$ ratio. They began by asserting that

When running the ML [meta learning] model initial values as well as scaling constants must be assigned. The initial values eliminate transients, which represent features of the model that are neither essential nor lasting. The initial value for positivity/negativity is 16 . The constants are used to scale the data, namely to be able to see the dynamics more clearly. (Losada \& Heaphy, 2004, p. 757)

This paragraph repeats the incorrect statements about the initial conditions, which as we have seen play no role in determining the long-time behavior of the system (namely, the features that are "essential" and "lasting").10 Therefore, pace Losada and Heaphy, a valid equation concerning the long-time behavior cannot involve the initial conditions. Furthermore, the constants $\sigma, b$, and $r$ are simply parameters in the Lorenz system; there is no

${ }^{10}$ Let us observe once again (see Footnote 7 above) that transient behavior is not necessarily without interest. But Losada and Heaphy (2004, p. 757) have here stressed that their own interest was focused on the long-time behavior, not the transients. 
reason why they should be "used to scale the data." But no matter: after drawing attention to the constant $b=8 / 3$, Losada and Heaphy completed their "reasoning" as follows:

With this background information, we can now calculate the $\mathrm{P} / \mathrm{N}$ ratio. To derive the $\mathrm{P} / \mathrm{N}$ ratio from the attractor's foci, we subtract the initial value and multiply it by the inverse of the scaling constant (0.375). For example, for high performance teams, we start with 31 , subtract 16 , and multiply by 0.375 . The result is 5.625 , which is very close to 5.614 , the result obtained by looking at the original time series data. We can now introduce the equation that allows us to calculate the positivity to negativity ratio $(\mathrm{P} / \mathrm{N})$ from emotional space $(\mathrm{E})$ :

$$
\mathrm{P} / \mathrm{N}=(E-i) b^{-1}
$$

where $E$ is emotional space, $i$ is the initial value of the positivity/negativity state variable (equal to 16), and $b^{-1}$ is the inverse scaling constant (equal to 0.375 ). If we apply this formula to the $E$ numbers for medium (21) and low-performance teams (17), we obtain results that are equally close to the ones obtained by looking directly at the time series data, thus further validating the ML model ... (Losada \& Heaphy, 2004, p. 757)

Indeed, the correspondence between data and theory is astounding (Losada \& Heaphy, 2004, p. 758 , Table 2):

$\begin{array}{lcc}\text { Positivity/Negativity Ratios from Time Series and Model } \\ & \text { Time Series Data } & \text { Model Data } \\ \text { High-performance teams } & 5.614 & 5.625 \\ \text { Medium-performance teams } & 1.855 & 1.875 \\ \text { Low-performance teams } & 0.363 & 0.375\end{array}$

And yet, the manipulations leading to the key equation $\mathrm{P} / \mathrm{N}=(E-i) b^{-1}$ are completely arbitrary. This whole exercise seems to be a post hoc "justification," with the artificial offset and scaling being applied to the model merely to allow it to produce "predictions" that resemble the observed data.

\section{Linking "connectivity" and the positivity-negativity ratio}

Combining the "results" of the preceding two sections, Losada and Heaphy (2004) deduced the desired connection between "connectivity" and the $\mathrm{P} / \mathrm{N}$ ratio: 


$$
\mathrm{P} / \mathrm{N}=(c-i-1) b^{-1}
$$

where $\mathrm{P} / \mathrm{N}$ is the ratio of positivity to negativity, $c$ is connectivity defined by the number of nexi, $i$ is the initial value of the positivity/negativity state variable and $b^{-1}$ is the inverse scaling constant. (Losada \& Heaphy, 2004, p. 758)

This final step is, of course, perfectly correct algebra, if one grants what has come before.

\section{Conclusion}

We confess that we have been unable to identify any mathematically or psychologically meaningful reasoning or analysis in Losada and Heaphy's (2004) derivation of their main result, $\mathrm{P} / \mathrm{N}=(c-i-1) b^{-1}$. As in Losada (1999), there are plenty of mathematical formulae - albeit here elementary algebra rather than nonlinear differential equations - but their only function, as far as we can tell, is to create, without any apparent justification, an equation that purports to describe a relationship between the $\mathrm{P} / \mathrm{N}$ ratio and "connectivity" and that happens to provide a good straight-line fit to three data points.

\section{Analysis of Fredrickson and Losada (2005)}

Fredrickson and Losada's (2005) central claim - and their key innovation beyond Losada (1999) and Losada and Heaphy (2004) — was the purported existence of a critical minimum positivity ratio value of 2.9013. Fredrickson and Losada took over the "results" of the two earlier articles as faits accomplis, with little or no explanation of the logic by which they were allegedly deduced. This seems to us a rather grave omission in an article that makes such extraordinary theoretical claims. Nevertheless, we once again invite the reader to assume for the sake of argument that the two preceding articles are correct in every detail; in this way we can highlight the independent flaws in Fredrickson and Losada (2005).

\section{Derivation of the critical minimum positivity ratio 2.9013}

Fredrickson and Losada (2005), after briefly recounting some studies suggesting that "high ratios of positive to negative affect would distinguish individuals who flourish from those who do not" (p. 680), then went on to assert that their more radical contention 
that "individuals or groups must meet or surpass a specific positivity ratio to flourish" is supported by "a nonlinear dynamics model empirically validated by Losada (1999)" (p. 681). After briefly summarizing Losada's (1999) experimental setup, they purported to explain the logic of Losada's work as follows:

Observation of the structural characteristics (i.e., amplitude, frequency, and phase) of the time series of the empirical data for these three performance categories led Losada to write a set of coupled differential equations to match each of the structural characteristics of the empirical time series. Table 1 presents these equations [which are the Lorenz equations but with $Y$ and $Z$ interchanged]. Model-generated time series were subsequently matched to the empirical time series by the inverse Fourier transform of the cross-spectral density function, also known as the cross-correlation function. Goodness of fit between the mathematical model and the empirical data was indicated by the statistical probability of the cross-correlation function at $p<.01$. (Fredrickson \& Losada, 2005, p. 681)

The trouble is, no such statistical matching between the model-generated time series and the empirical time series is anywhere even alluded to - much less presented - in Losada (1999). (Indeed, as noted earlier, no empirical data for the variables $X, Y, Z$ are presented at all.) Nor, for that matter, is any such matching mentioned anywhere in the later article of Losada and Heaphy (2004). The alleged empirical validation of Losada's mathematical model is thus unsupported by anything in the three subject articles. (Let us therefore put aside the otherwise crucial question of precisely what statistical procedures were employed to perform this matching, and whether they are valid.)

Fredrickson and Losada (2005) then went on to present the famous "butterfly" plots showing the trajectories of the Lorenz system (p. 682, Figure 1). They asserted that the three plots are, respectively, "derived from the empirical time series of the flourishing, high-performance teams" (p. 681), "derived from the empirical time series of the medium-performance teams" (p. 681), and "derived from the empirical time series of the low-performance teams" (p. 682) - they repeated this phrasing verbatim three times - but they provided no evidence that 
these claims are true (neither, as we saw, did Losada, 1999). Figure 1 shows the results of computer simulations of the Lorenz equations, nothing more.

Finally, Fredrickson and Losada (2005) derived the ideal minimum positivity ratio of 2.9013 by recalling (pp. 682-683) that

Subsequent work on the model (Losada \& Heaphy, 2004) revealed that the positivity ratio relates directly to the control parameter by the equation $P / N=\left(c-Y_{0}-1\right) b^{-1} \ldots$ Past mathematical work on Lorenz systems by Sparrow (1982) and others (Frøyland \& Alfsen, 1984; Michielin \& Phillipson, 1997) has established that when $r$, the control parameter in the Lorenz model, reaches 24.7368 , the trajectory in phase space shows a chaotic attractor. Losada (1999) established the equivalence between his control parameter, $c$, and the Lorenzian control parameter, $r$. Using the above equation, it is known that the positivity ratio equivalent to $r=24.7368$ is 2.9013 .

Fredrickson and Losada did not explain where $r_{\text {crit }}=24.7368$ comes from or exactly how it leads to $(P / N)_{\text {crit }}=2.9013$, but we can fill in the logic of their derivation (making explicit the missing hypotheses) and learn something new in the process. The first step is to accept uncritically the main "result" of Losada and Heaphy (2004), namely, $P / N=(c-i-1) b^{-1}$ (here Fredrickson and Losada have renamed $i$ as $Y_{0}$ ). The second step is to accept that Losada (1999) "established" the equivalence of $c$ and $r$ (although in fact he merely declared it by fiat, with curious consequences that we have already noted). We now set $c$ equal to the value $r_{\text {crit }}=\sigma(\sigma+b+3) /(\sigma-b-1)$ that constitutes the boundary between nonchaotic and chaotic behavior in the Lorenz system. (When $\sigma=10$ and $b=8 / 3$, this yields $r_{\text {crit }}=470 / 19 \approx 24.7368$.) Simple algebra then gives the final formula

$$
(P / N)_{\text {crit }}=\frac{\sigma(\sigma+b+3)}{b(\sigma-b-1)}-\frac{i+1}{b} .
$$

Specializing to $\sigma=10, b=8 / 3$ and $i=16$, we obtain

$$
(P / N)_{\text {crit }}=\frac{441}{152}=2.901 \overline{315789473684210526}
$$

where - denotes an infinitely repeating decimal. Fredrickson and Losada (2005) were 
therefore far too modest to claim only five significant digits; their critical positivity ratio is in fact an exact rational number!

Unfortunately, there is one final, yet crucial, flaw lurking here: the values of $\sigma, b$, and (especially) $i$ plugged into Equation [6 are totally arbitrary, at least within wide limits; so the predicted critical positivity ratio is totally arbitrary as well. Choose different values of

the parameters $\sigma, b, i$ and one gets a completely different prediction for $(P / N)_{\text {crit }}$. Recall that Saltzman (1962) chose $\sigma=10$ for illustrative purposes and purely for convenience; then Lorenz (1963) and Losada (1999) followed him. Were humans to have eight fingers on each hand instead of five, Saltzman, and in turn presumably Lorenz and Losada, might well have chosen $\sigma=16$ instead of $\sigma=10$ - which (with $b=8 / 3$ ) produces a very similar Lorenz attractor, except that the borderline of chaos is now $r_{\text {crit }}=1040 / 37=28 . \overline{108}$, and the predicted critical positivity ratio ( with $i=16)$ is $(P / N)_{\text {crit }}=1233 / 296=4.1655 \overline{405}$. Yet other values of $\sigma, b, i$ would yield still different predictions for $(P / N)_{\text {crit }}$.

Thus, even if one were to accept for the sake of argument that every single claim made in Losada (1999) and Losada and Heaphy (2004) is correct, and even if one were to further accept that the Lorenz equations provide a valid and universal way of modeling human emotions, then the ideal minimum positivity ratio that Fredrickson and Losada (2005) claimed to have derived from Losada's "empirically validated" nonlinear-dynamics model would still be nothing more than an artifact of the arbitrary choice of an illustratively convenient value made by a geophysicist in Hartford in 1962 .

\section{Fredrickson and Losada's empirical study}

In view of the foregoing vitiation of Fredrickson and Losada's (2005) "derivation" of the ideal minimum positivity ratio 2.9013, a deep analysis of the results from their empirical study would be superfluous. The fact that "flourishing" college students exhibited higher average positivity ratios (3.2) than those who were "languishing" (2.3) should not come as a surprise; there is nothing inherently implausible about the idea that people with a higher ratio of positive to negative emotions might experience better outcomes than those with a lower ratio. But the suggestion that people with a positivity ratio of 2.91 are in some 
discontinuous way significantly better off than those with a ratio of 2.90 , simply because this number has crossed some magic line, is not supported by any evidence.

\section{An upper critical positivity ratio?}

Compared to the abuse of mathematics in the theoretical part of their article, Fredrickson and Losada's (2005) study of "flourishing" versus "languishing" college students does at least provide some empirical evidence that a higher positivity ratio typically corresponds to better outcomes than a lower one. By contrast, their claim that there is also a precise desirable upper limit to the positivity ratio does not appear to be supported by any evidence whatsoever. Their prediction (p. 684) of an upper limit of 11.6346 is based entirely on the purported correspondence between human emotions and the Lorenz system, combined with a gross mathematical error concerning the latter. More precisely, Fredrickson and Losada equated psychological "flourishing" with the "complex dynamics" of the chaotic Lorenz attractor (pp. 682, 684); therefore, they (apparently) reasoned, if there is a value of the control parameter $r$ beyond which chaos no longer occurs, then the end of chaotic attraction means the end of flourishing - in this case, at a positivity-ratio value that the authors "estimate" to be 11.6346 (p. 684). Assuming that they arrived at this value using the same calculation method as for the ideal minimum positivity ratio, this corresponds to $r \approx 48.0256$. However, it is not the case that chaotic attraction in the Lorenz system (with $\sigma=10$ and $b=8 / 3$ ) disappears beyond this value of $r$. Rather, within the regime $r>r_{\text {crit }} \approx 24.7368$ there is an intricate pattern of windows of periodicity (many of them quite narrow) alternating or mixed with chaotic behavior, until at least $r \approx 200$ (Sparrow, 1982, Chapters 4 and 5, see especially Figure 5.12); in particular, one extremely narrow periodic window appears to lie at $48.0259 \lesssim r \lesssim 48.0271$ (Frøyland \& Alfsen, 1984; Fang \& Hao, 1996). Thus, if one were to take seriously the reasoning of Fredrickson and Losada, there would exist a complicated sequence of "undesirable" positivity-ratio intervals lying within the mostly-desirable range above 2.9013 .

But it goes without saying that this prediction is moot, because, as we have demonstrated throughout the present paper, the alleged connection between human emotions and the 
Lorenz equations is entirely fanciful.

\section{Conclusion}

Fredrickson and Losada (2005) in effect claimed - on the basis of an analysis of verbal statements made in a series of one-hour meetings held in a laboratory setting by business teams of exactly eight people, combined with some solemn invocations of the Lorenz equations - to have discovered a universal truth about human emotions, valid for individuals, couples, and groups of arbitrary size and capable of being expressed numerically to five significant digits. This claim - which was presented with no qualification or discussion of possible limits to its validity - would, if verified, surely require much of contemporary psychology and neuroscience to be rewritten; purely on that basis we are surprised that, apparently, no researchers have critically questioned this claim, or the reasoning on which it was based, until now.

We do not here call into question the idea that positive emotions are more likely to build resilience than negative emotions, or that a higher positivity ratio is ordinarily more desirable than a lower one. But to suggest that some form of discontinuity sets in at some special value of the positivity ratio - especially one that is independent of all demographic and cultural factors - seems far-fetched. We cannot, of course, prove that no such "tipping point" exists; but we believe that we have adequately demonstrated here that even if it does, Fredrickson and Losada's (2005) article - based on a series of erroneous and, for the most part, completely illusory "applications" of mathematics - has not moved science any nearer to finding it.

Fredrickson and Losada (2005, p. 685) concluded their article by observing modestly that "Our discovery of the critical 2.9 positivity ratio may represent a breakthrough." Would that it were so.

\section{Concluding remarks}

The process that has taken place in this trio of articles was presciently foreseen four decades ago by the sociologist Stanislav Andreski: 
The recipe for authorship in this line of business is as simple as it is rewarding: just get hold of a textbook of mathematics, copy the less complicated parts, put in some references to the literature in one or two branches of the social studies without worrying unduly about whether the formulae which you wrote down have any bearing on the real human actions, and give your product a good-sounding title, which suggests that you have found a key to an exact science of collective behaviour. (Andreski, 1972, pp. 129-130)

To be sure, Andreski's acerbic description of pseudoscientific work in the social sciences may seem an exaggeration, and in most cases it probably is. But as applied to the articles of Losada (1999), Losada and Heaphy (2004), and Fredrickson and Losada (2005), Andreski's portrayal is, alas, literally accurate.

Let us stress that our concern here is with the objective properties of published texts, not the subjective states of mind of the authors (which might, however, be of interest to philosophers, such as Frankfurt, 2005). We do not, for example, have an opinion about the degree to which excessive enthusiasm, sincere self-deception, or other motivations may have influenced Losada and colleagues when writing their articles. Our only interest here is to bring the fundamental errors in this widely-cited body of work to the attention of the scientific community, before its considerable influence can do any further damage to the cause of science in general and academic psychology in particular.

More generally, one of our aims in writing this article has been to alert scholars who may be considering the use of differential-equation models in their work to the need to ensure that their application of differential equations to a specific natural or social phenomenon has been adequately justified, be it by theoretical arguments or by empirical evidence or both. To this end, we modestly propose that our checklist of criteria VA1-VA5 for the valid application of differential equations might be a useful tool. In any case, we anticipate that the publication of the present paper may stimulate a lively debate on this subject.

We wish to conclude on an optimistic note. The fundamental property of science, that it self-corrects and recovers from errors, is often touted - and rightly so — as a strength that 
distinguishes it from pseudoscience. Unfortunately, in psychology just as in other scientific disciplines, things do not always work out that way, at least in the short run. We are therefore grateful for the opportunity to publish this critique; we hope that other scholars will be encouraged to question, in public fora such as this one, other research that seems to require correction.

\section{References}

Andreski, S. (1972). Social sciences as sorcery. London, UK: Andre Deutsch.

Bales, R. F. (1950). Interaction process analysis: A method for the study of small groups. Cambridge, MA: Addison-Wesley.

Bradburn, N. M. (1969). The structure of psychological well-being. Chicago, IL: Aldine.

Fang, H.-P., \& Hao, B.-L. (1996). Symbolic dynamics of the Lorenz equations. Chaos, Solitons \& Fractals, 7(2), 217-246. doi:10.1016/0960-0779(95)00046-1

Frankfurt, H. G. (2005). On bullshit. Princeton, NJ: Princeton University Press.

Fredrickson, B. L. (1998). What good are positive emotions? Review of General Psychology, 2(3), 300-319. doi:10.1037/1089-2680.2.3.300

Fredrickson, B. L. (2001). The role of positive emotions in positive psychology: The broaden-andbuild theory of positive emotions. American Psychologist, 56 (3), 218-226. doi:10.1037/0003066X.56.3.218

Fredrickson, B. L. (2004). The broaden-and-build theory of positive emotions. Philosophical Transactions of the Royal Society of London, Series B: Biological Sciences, 359(1449), 1367-1377. doi:10.1098/rstb.2004.1512

Fredrickson, B. L. (2009). Positivity: Groundbreaking research reveals how to embrace the hidden strength of positive emotions, overcome negativity, and thrive. New York, NY: Crown. Published in paperback as Positivity: Top-notch research reveals the 3-to-1 ratio that will change your life.

Fredrickson, B. L., \& Kurtz, L. E. (2011). Cultivating positive emotions to enhance human flourishing. In S. I. Donaldson, M. Csikszentmihalyi, \& J. Nakamura (Eds.), Applied positive psychology: Improving everyday life, health, schools, work, and society (pp. 35-48). New York, NY: Routledge. 
Fredrickson, B. L., \& Losada, M.F. (2005). Positive affect and the complex dynamics of human flourishing. American Psychologist, 60(7), 678-686. doi:10.1037/0003-066X.60.7.678

Frøyland, J., \& Alfsen, K. H. (1984). Lyapunov-exponent spectra for the Lorenz model. Physical Review A, 29(5), 2928-2931. doi:10.1103/PhysRevA.29.2928

Hilborn, R. C. (2000). Chaos and nonlinear dynamics: An introduction for scientists and engineers. New York, NY: Oxford University Press.

Kellert, S. H. (1995). When is the economy not like the weather? The problem of extending chaos theory to the social sciences. In A. Albert (Ed.), Chaos and society (pp. 35-47). Amsterdam, Netherlands: IOS Press.

Lorenz, E. N. (1963). Deterministic nonperiodic flow. Journal of the Atmospheric Sciences, 20(2), 130-141. doi:10.1175/1520-0469(1963)020<0130:DNF>2.0.CO;2

Lorenz, E. N. (1993). The essence of chaos. Seattle, WA: University of Washington Press.

Losada, M. (1999). The complex dynamics of high performance teams. Mathematical and Computer Modelling, 30(9-10), 179-192. doi:10.1016/S0895-7177(99)00189-2

Losada, M., \& Heaphy, E. (2004). The role of positivity and connectivity in the performance of business teams: A nonlinear dynamics model. American Behavioral Scientist, 47(6), 740-765. doi:10.1177/0002764203260208

Losada, M., \& Markovitch, S. (1990). GroupAnalyzer: A system for dynamic analysis of group interaction. In Proceedings of the 23rd Hawaii international conference on system sciences (pp. 101-110). Los Alamitos, CA: IEEE Computer Society Press. doi:10.1109/HICSS.1990.205245

Losada, M., Sánchez, P., \& Noble, E. E. (1990). Collaborative technology and group process feedback: Their impact on interactive sequences in meetings. In CSCW '90 - Proceedings of the 1990 ACM conference on computer-supported cooperative work (pp. 53-64). New York, NY: Association for Computing Machinery. doi:10.1145/99332.99341

Luoma, J., Hämäläinen, R. P., \& Saarinen, E. (2008). Perspectives on team dynamics: Meta learning and systems intelligence. Systems Research and Behavioral Science, 25(6), 757767. doi:10.1002/sres.905

Michielin, O., \& Phillipson, P. E. (1997). Map dynamics study of the Lorenz equations. International Journal of Bifurcation and Chaos, 7, 373-382. doi:10.1142/S0218127497000248 
Ruelle, D. (1994). Where can one hope to profitably apply the ideas of chaos? Physics Today, $47(7), 24-30$. doi:10.1063/1.881395

Saltzman, B. (1962). Finite amplitude free convection as an initial value problem-I. Journal of the Atmospheric Sciences, 19(4), 329-341. doi:10.1175/1520-0469(1962)019<0329:FAFCAA >2.0.CO;2

Seligman, M. E. P. (2011a). Flourish: A visionary new understanding of happiness and well-being. New York, NY: Free Press.

Seligman, M. E. P. (2011b, July 6). Lecture to the Royal Society for the Arts [Video file]. Retrieved from http://www.thersa.org/events/video/vision-videos/martin-seligman. See 02:55-03:50.

Sokal, A., \& Bricmont, J. (1998). Fashionable nonsense: Postmodern intellectuals' abuse of science. New York, NY: Picador USA. Published in the UK as Intellectual impostures: Postmodern philosophers' abuse of science. London, UK: Profile Books.

Sparrow, C. (1982). The Lorenz equations: Bifurcations, chaos, and strange attractors. New York, NY: Springer-Verlag.

Stewart, I. (1997). Does God play dice?: The new mathematics of chaos. London, UK: Penguin.

Strogatz, S. H. (1994). Nonlinear dynamics and chaos: With applications to physics, biology, chemistry, and engineering. Reading, MA: Addison-Wesley.

Williams, G. P. (1997). Chaos theory tamed. Washington, DC: Joseph Henry Press. 